\title{
Cultured thymus tissue implantation promotes donor-specific tolerance to allogeneic heart transplants
}

\author{
Jean Kwun, ${ }^{1}$ Jie Li, ${ }^{1}$ Clay Rouse, ${ }^{2}$ Jae Berm Park, ${ }^{1}$ Alton B. Farris, ${ }^{3}$ Maragatha Kuchibhatla, ${ }^{4}$ \\ Joseph W. Turek, ${ }^{1}$ Stuart J. Knechtle, ${ }^{1}$ Allan D. Kirk, ${ }^{1}$ and M. Louise Markert ${ }^{5,6}$ \\ 'Department of Surgery, Duke University Medical Center, Durham, North Carolina, USA. 'Division of Laboratory Animal \\ Resources, Duke University, Durham, North Carolina, USA. ${ }^{3}$ Department of Pathology, Emory University, Atlanta, Ceorgia, \\ USA. ${ }^{4}$ Department of Biostatistics and Bioinformatics, ${ }^{5}$ Department of Immunology, and ${ }^{6}$ Department of Pediatrics, Duke \\ University Medical Center, Durham, North Carolina, USA.
}

Eighty-six infants born without a thymus have been treated with allogeneic cultured thymus tissue implantation (CTTI). These infants, who lack T cells and are profoundly immunodeficient at birth, after CTTI from an unmatched donor develop T cells similar to those of recipient that are tolerant to both their own major histocompatibility antigens and those of the donor. We tested use of CTTI with the goal of inducing tolerance to unmatched heart transplants in immunocompetent rats. We thymectomized and T cell-depleted Lewis rats. The rats were then given cultured thymus tissue from F1 (Lewis $\times$ Dark Agouti ) under the kidney capsule and vascularized Dark Agouti (DA) heart transplants in the abdomen. Cyclosporine was administered for $\mathbf{4}$ months. The control group did not receive CTTI. Recipients with CTTI showed repopulation of naive and recent thymic emigrant CD4 T cells; controls had none. Recipients of CTTI did not reject DA cardiac allografts. Control animals did not reject DA grafts, due to lack of functional T cells. To confirm donor-specific unresponsiveness, MHC-mismatched Brown Norway (BN) hearts were transplanted 6 months after the initial DA heart transplant. LW rats with LWXDA CTTI rejected the third-party BN hearts (mean survival time 10 days); controls did not. CTTI recipients produced antibody against third-party BN donor but not against the DA thymus donor, demonstrating humoral donor-specific tolerance. Taken together, F1(LWxDA) CTTI given to Lewis rats resulted in specific tolerance to the allogeneic DA MHC expressed in the donor thymus, with resulting long-term survival of DA heart transplants after withdrawal of all immunosuppression.

Conflict of interest: Funding was obtained for this study in part from Enzyvant. MLM developed the technology of thymus transplantation, which has been licensed to Enzyvant. MLM has received royalties from Enzyvant. Portions of MLM's and her research team's salaries are being paid by the funding from Enzyvant. If the technology is commercially successful in the future, MLM and Duke may benefit financially.

Copyright: (ㄷ) 2020, American Society for Clinical Investigation.

Submitted: May 1, 2019

Accepted: April 23, 2020

Published: June 4, 2020

Reference information: /CI Insight. 2020;5(11):e129983.

https://doi.org/10.1172/jci.

insight.129983.

\section{Introduction}

Ray Owen first observed immune tolerance to other individuals' blood in Freemartin calves (1), and later Peter Medawar was the first to induce transplant tolerance by injecting neonatal mice with adult donor tissue/cell suspension (2). These examples of tolerance induction were related to the immature nature of the neonatal immune system $(3,4)$. Later, the central role of T cells in transplant rejection was identified (5, 6), and deletion of developing alloreactive T cells in the host thymus was thought to be the primary mechanism of tolerance induction $(7,8)$. There were many attempts to promote tolerance to allogeneic organs by introducing thymus composite tissues or vascularized thymus in which the thymus expressed donor MHC in miniature swine (9-12). The thymoheart was formed by injecting finely minced thymus tissue of the donor type into the atrial appendages of miniature swine. Those hearts were able to survive in an allogeneic recipient for up to approximately 194 days (10). A similar procedure was performed for the thymokidney (9). Tolerance was observed in the miniature swine that received a thymokidney and were followed for up to 60 days. Another study transplanted allogeneic vascularized donor thymic lobes and donor hearts at the same time (12). Allograft survival in the miniature swine was followed up to 301 days when the last animal was sacrificed. Despite success for up to 300 days, data have not been published showing the long-term success needed for human transplantation.

Experimental allogeneic cultured thymus tissue implantation (CTTI) used in 86 athymic infants with complete DiGeorge anomaly resulted in development of naive T cells in the 61 survivors $(71 \%)(13,14)$. 
Almost $30 \%$ of the infants died from preexisting conditions and infections. Studies of tolerance induction in survivors after CTTI from 1993 to 2018 using mixed lymphocyte reactions showed development of tolerance to the alloantigens of the thymus donor in the athymic infants (14). One example of tolerance induction to a solid organ occurred in an infant with complete DiGeorge anomaly who had profound hypoparathyroidism. The infant was treated with CTTI plus parental parathyroid. The CTTI expressed the class II antigens of the parent that were not inherited by the infant (15). The CTTI did not express the 3 mismatched parental class I alleles. The parental parathyroid functioned for 10 years with no immune suppression until the patient was given a measles/mumps/rubella vaccine. The parental parathyroid was rejected within 2 weeks and the patient had to return to calcium replacement. The mechanism for the loss of parathyroid function was likely rejection of the parental parathyroid by recipient CD8 T cells, one-third of which are inherently alloreactive and would have recognized the parental unmatched class I antigens as foreign. If the CTTI had expressed the uninherited class I antigens, the CD8 T cells developing in the thymus would have been deleted if they reacted against the uninherited class I antigens, and the patient would almost certainly still have parathyroid function.

Several other findings in recipients of CTTI should be mentioned. Recipients of CTTI were able to control viral infections such as Epstein-Barr virus that would have been fatal before CTTI (16). In addition, these infants, who had essentially no naive T cells before CTTI, developed naive T cells approximately 6 months after CTTI $(13,17)$. Biopsies of the transplanted cultured thymus tissue have demonstrated thymopoiesis on IHC (18). Flow cytometry and spectratyping have shown development of a diverse $\mathrm{T}$ cell repertoire. Based on the human data showing tolerance to unmatched thymus MHC antigens, we evaluated CTTI, with the same methods used clinically, for its ability to induce donor-specific tolerance in a rat heart transplantation model. Our studies show that transplanting unmatched hearts along with donor CTTI expressing the heart donor's MHC class I and class II antigens induces tolerance to the antigens of the donor heart while preserving alloreactivity toward other MHC antigens.

\section{Results}

In vitro thymic cultures, in vivo engraftment. In order to perform allogeneic CTTI in a rat model with procedures used for the treatment of athymic infants with complete DiGeorge anomaly, thymus was harvested from 3-day-old F1(Lewis $\times$ Dark Agouti [LWxDA]) rat pups. Each postnatal thymus was cut into 4 pieces and cultured for 5 to 7 days in thymus organ medium (Supplemental Figure 1; see also methods; supplemental material available online with this article; https://doi.org/10.1172/jci.insight.129983DS1). Histologic analysis (Supplemental Figure 2) showed reduced $\mathrm{Ki}_{67}{ }^{+} \mathrm{CD}^{+}$cells in the thymus after culture, similar to the change seen after culture of thymus tissue used for patients (18). As in cultured human thymus, the network of thymic epithelial cells (TECs) was preserved in the rat cultured thymus tissue based on cytokeratin (CK) staining (Supplemental Figure 2). The thymus, after being in culture for 5-7 days, was implanted under the kidney capsule of a Lewis (LW) rat at the same time as the vascularized Dark Agouti (DA) heart is transplanted into the abdomen. As shown in Figure 1A, recipients were thymectomized and treated with anti-CD5 mAb (OX19; $1 \mathrm{mg}$, i.p.) every 5 days for 3 doses. The recipients also received cyclosporine A (CsA), approximately $2.5 \mathrm{mg} / \mathrm{kg} / \mathrm{d}$ after thymus transplantation using osmotic pumps. CsA was discontinued 4 months after thymus transplantation when the test group had naive T cells over $10 \%$. At 6-7 months, the third-party Brown Norway (BN) heart was transplanted in the neck. The thymus graft and all hearts were evaluated at necroscopy 8 months after CTTI when the test group rejected the cervical $\mathrm{BN}$ heart. As predicted, recipient-derived LW T cells, not expressing DA MHC, appeared in the peripheral blood of thymus transplant recipients (Supplemental Figure 3B). We evaluated circulating T cell subpopulations as well as non-T cell populations (B cell and NK cell) via flow cytometry (Figure 1B and Supplemental Figure 4). Gating strategies for each subpopulation are shown in Supplemental Figure 5. We predicted that the LW T cells would be tolerant to the DA antigen of the DA heart because the LW T cells had developed in an LWxDA thymus (Figure 1A). Total circulating CD3 T cell numbers were not significantly different in between groups before transplantation. As expected, LW recipients with CTTI showed significantly increased numbers of circulating CD4 and CD8 T cells compared with control animals without CTTI after transplantation (Figure 1B). Furthermore, animals with CTTI showed significantly increased repopulation of naive $\left(\mathrm{CD} 62 \mathrm{~L}^{+} \mathrm{CD} 45 \mathrm{RC}^{+}\right) \mathrm{CD} 4$ and $\mathrm{CD} 8 \mathrm{~T}$ cells as well as recent thymic emigrant (RTE) $\left(\mathrm{CD}^{+} \mathrm{C}^{+} \mathrm{CD} 45 \mathrm{RC}^{-}\right) \mathrm{T}$ cells in the peripheral blood, whereas control groups without thymus transplantation showed low level of circulating naive CD4 and CD8 T cells and did not show circulating RTE CD4 and 
A
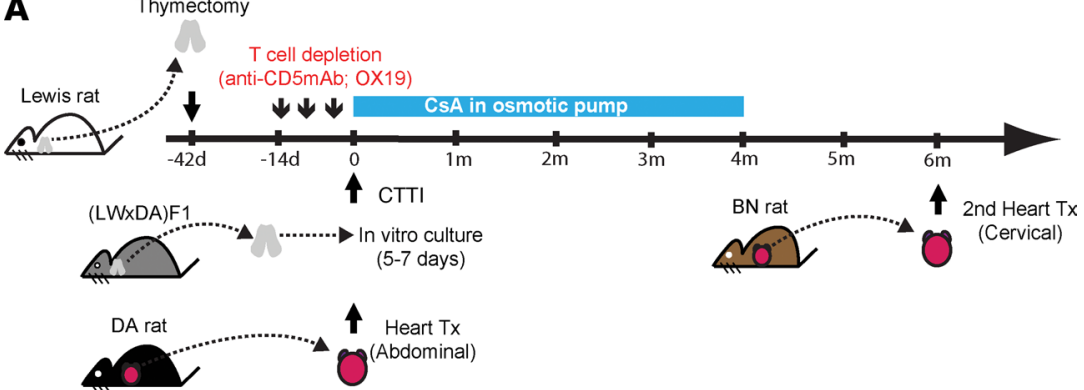

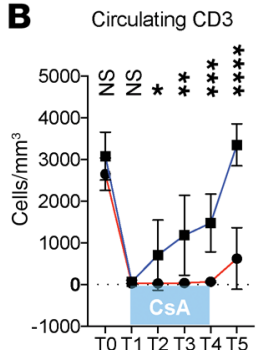

Control (w/o CTTI) - With CTTI

\begin{tabular}{|l|}
\hline T0: Pre-depletion \\
T1: Post-depletion \\
T2: 2 mon post-depletion \\
T3: 3 mon post-depletion \\
T4: 4 mon post-depletion \\
T5: 6 mon post-depletion \\
\hline
\end{tabular}

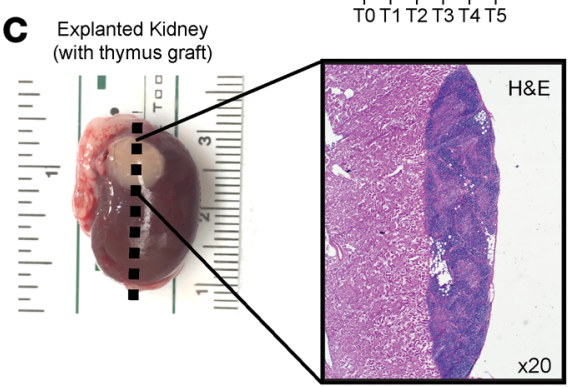

CD4 T cells

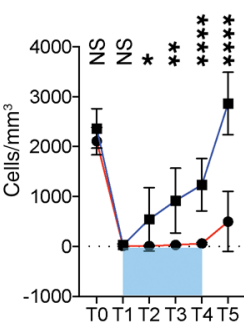

CD8 T cells

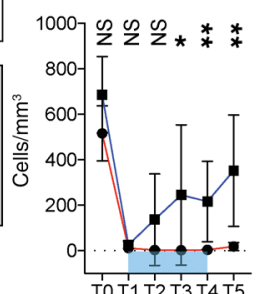

\&E

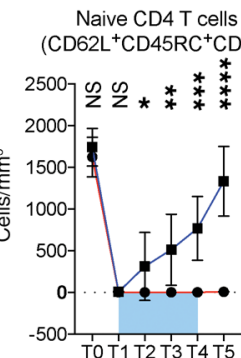

Naive CD8 T cells $\left(\mathrm{CD}_{2} \mathrm{~L}^{+} \mathrm{CD} 45 \mathrm{RC}^{+} \mathrm{CD}^{+}\right)$

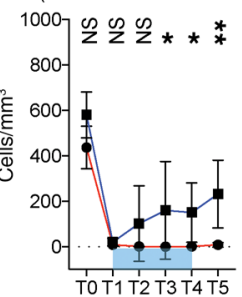

CD4 RTE

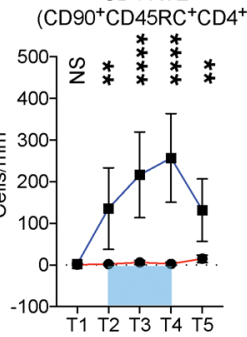

CD8 RTE $\left(\mathrm{CD}^{+} \mathrm{CD}^{+} 5 \mathrm{RC}^{+} \mathrm{CD}^{+}\right)$
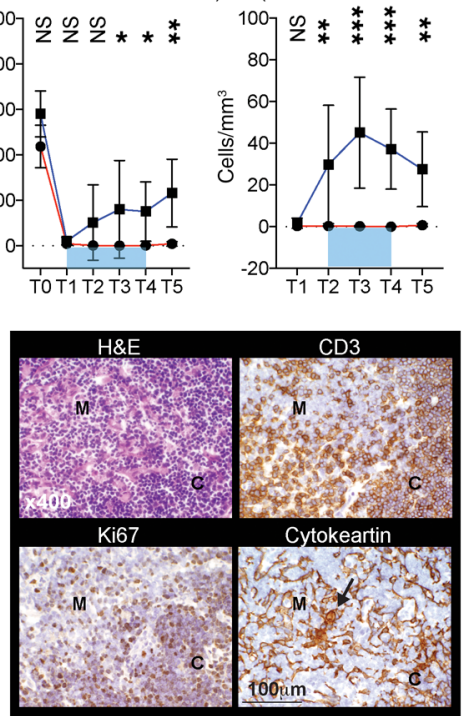

Figure 1. T cell reconstruction and functional transplanted thymus tissue in cardiac allograft recipients with CTTI. (A) Schematic outline of experimental design to show naive T cell reconstitution, thymopoiesis, and donor-specific tolerance induced by CTTI. All LW rats were thymectomized and T cell depleted via anti-CD5 mAb before heart transplantation and CTTI. CTTI from F1(LWxDA) rats and hearts from DA rats were transplanted into thymectomized LW recipients. CsA was given for 4 months after transplantation via osmotic pump. The third-party BN heart was transplanted into the neck 2 to 3 months after CsA discontinuation. Control rats experienced identical procedures except they did not receive CTTI. (B) Circulating $\mathrm{T}$ cell repopulation after $\mathrm{T}$ cell depletion and thymus and heart transplantation. All animals showed dramatic reduction of circulating T cells after T cell depletion. Cardiac allograft recipients with CTTI (blue line) showed gradual repopulation of circulating T cells. Animals without CTTI also showed some degree of circulating T cells (red line). However, naive and recent thymic emigrants CD4 and CD8 T cells were significantly increased $(P<0.01)$ in animals with CTTI, whereas control animals showed neither circulating naive CD4 and CD8 T cells nor RTE CD4 and CD8 T cells. (C) Engrafted cultured thymus tissues under the renal capsule on day 180 in a recipient of cardiac allograft. Histology showed a distinct structure separate from renal tissue (original magnification, $\times 20$ ). Engrafted cultured thymus tissue (right panel) showed a normal thymus structure (H\&E), viable T cells (CD3), T cell proliferation (Ki67), and Hassall body formation (black arrow) with a lacy pattern (cytokeratin) on epithelial cells, confirming the viability of thymus with thymopoiesis (original magnification, $\times 200$ ). Data are presented as means \pm SD; $n=8-9$ animals per group; student's $t$ test, ${ }^{*} P<0.05 ;{ }^{* *} P<$ 0.01 ; ${ }^{* *} P<0.001,{ }^{* * * *} P<0.0001$; NS, not significant $(P>0.05)$. CTTI, cultured thymus tissue implantation; LW, Lewis; LWxDA, Lewis $\times$ Dark Agouti; DA, Dark Agouti; CsA, cyclosporine A; BN, Brown Norway; RTE, recent thymic emigrant. 


\section{A DA donor heart survival}

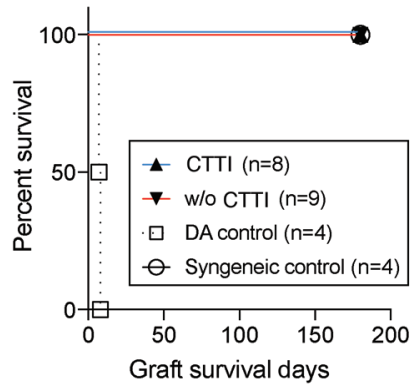

B

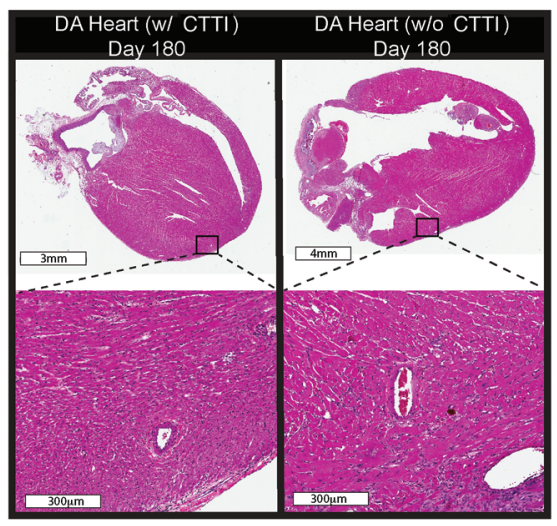

C

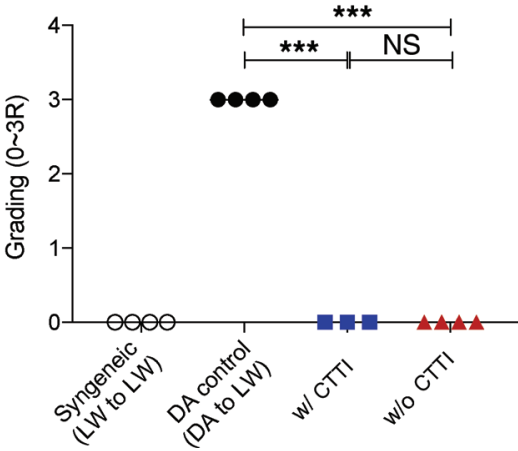

Figure 2. Long-term cardiac allograft survival regardless of thymus cotransplantation. (A) Graft survival of DA heart in recipients with or without CTTI. Kaplan-Meier survival curve showed significantly prolonged graft survival from animals with or without CTTI and syngeneic controls (LW heart into LW rat) compared with LW rats with DA heart transplants without immunosuppression/thymectomy (DA control). (B) Representative scanned image of explanted DA heart graft on day 180 from animals with and without CTTI. Images were adapted from whole slide scan. (C) ISHLT grading showed a significant reduction of rejection in both recipients with CTTI and without CTTI compared with DA control with no immunosuppression ( $n=3-4$ per group). Turkey test, ${ }^{* * *} P<0.001 ;$ NS, not significant $(P>0.05)$. DA, Dark Agouti; CTTI, cultured thymus tissue implantation; LW, Lewis; ISHLT, International Society for Heart and Lung Transplantation.

CD8 T cells (Figure 1B). In addition, immunohistologic analysis of transplanted cultured thymus tissue explanted on day 180 showed normal thymus histology, viable T cells (CD3), T cell proliferation (Ki67), and a lacy pattern of CK with Hassall body formation (arrow) on TECs from the CTTI (Figure 1C). These observations confirm the viability and function (thymopoiesis) of the transplanted thymus in the animals receiving allogeneic heart transplantation. Taken together, rats that received cardiac allografts with CTTI demonstrated thymopoiesis with naive $\mathrm{T}$ cell development.

No graft rejection with or without CTTI in thymectomized recipients. It was expected that $\mathrm{T}$ cells reactive to the DA donor would not develop since the T cells developed in CTTI express DA as well as LW. We evaluated the DA heart for evidence of rejection. As shown in Figure 2A, LW rats with DA heart transplants without any immunosuppressive treatment rejected the DA heart grafts within 10 days (the DA control). However, even after developing RTE $\left(\mathrm{CD} 90^{+} \mathrm{CD} 45 \mathrm{RC} \mathrm{C}^{-}\right) \mathrm{T}$ cells, LW recipients with CTTI did not reject (no cessation of beating) the DA cardiac allografts $(n=8)$. Unexpectedly, LW control animals without CTTI also did not reject the DA cardiac graft $(n=9)$. Both groups showed good beating quality for the entire study period (day 180). Since continuous graft beating does not necessarily imply absence of rejection, we sacrificed 2 recipients 2 months after cessation of immunosuppression (before third-party BN cervical heart transplantation) to confirm that there was no rejection. The explanted cardiac allografts (DA hearts) from both animals showed minimal mononuclear cell infiltration (Figure 2B) and with no signs of rejection by 2004 International Society for Heart and Lung Transplantation (ISHLT) grading (Figure 2C). Based on the reconstitution of naive T cells after CTTI, we believe that animals with CTTI lost their donor-reactive T cell repertoire, whereas animals without CTTI did not fully reconstitute their $\mathrm{T}$ cell populations (general hyporesponsiveness).

Alloreactivity against third-party vascularized heart transplantation. In order to confirm the donor-specific unresponsiveness (tolerance) versus general hyporesponsiveness, we performed additional fully MHC-mismatched BN heart transplantation in both groups of animals at 6-7 months (days 180-210) after DA heart transplantation (Figure 1A). As shown in Figure 3A, LW rats with CTTI rapidly rejected (cessation of graft beating) the third-party BN heart ( $n=5$, median survival time [MST] $=10 \pm 1.0$ days). However, the control LW animals without CTTI did not reject the third-party hearts (Figure 3A) $(n=6$, MST $\geq 38.5 \pm$ 8.9 days), possibly due to the lack of any alloreactive T cells. In accordance with the rejection of third-party heart by animals with CTTI and the lack of rejection of the third-party heart in animals without CTTI (Figure 3, A and B), histological analysis (Figure 3C) confirmed that animals with CTTI showed increased mononuclear cell infiltration in the heart allograft (Figure 3C, third image), whereas animals without CTTI showed a pristine BN heart allograft (Figure 3C, fourth image). It is also notable that the BN hearts in the recipients with CTTI were greatly enlarged (Figure 3C, third image, and Supplemental Figure 6, panel with CTTI), whereas the DA hearts were smaller than the native hearts (Supplemental Figure 6 panel without 
A

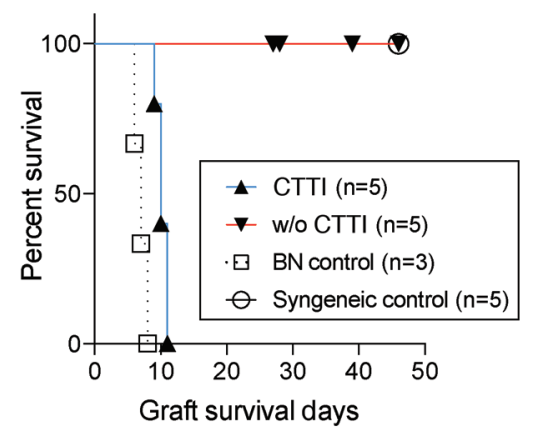

B

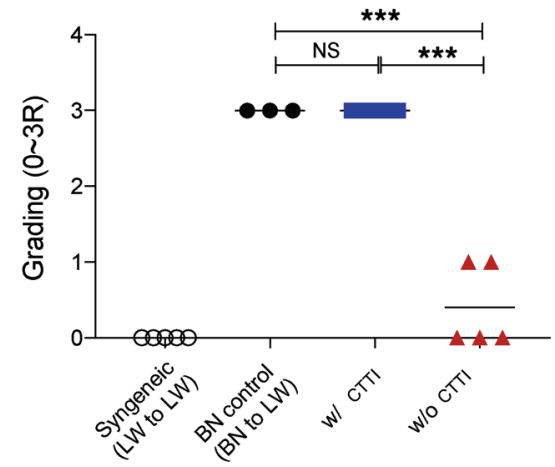

C

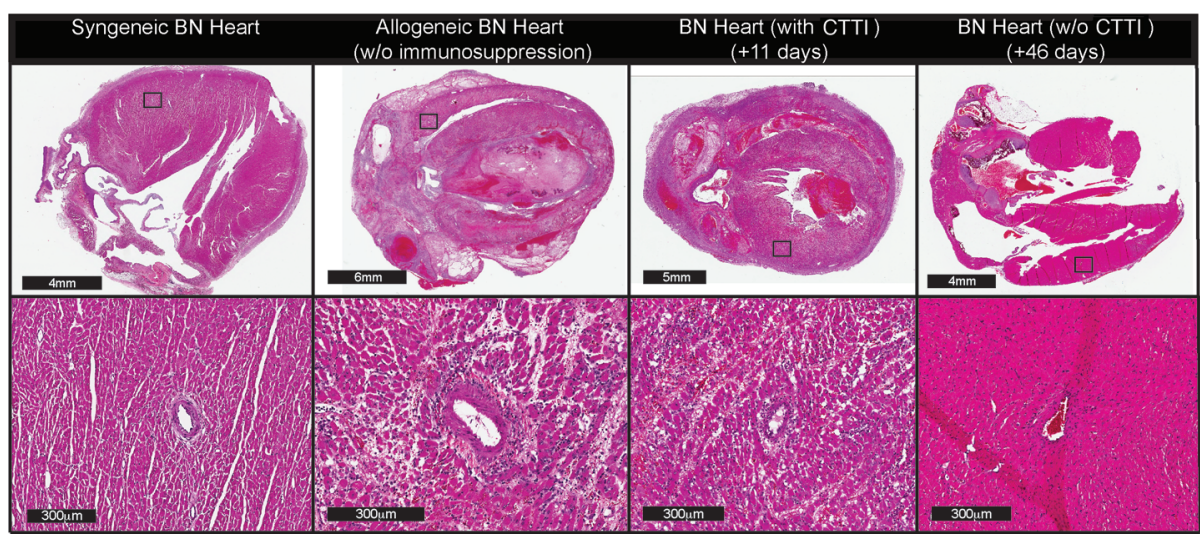

Figure 3. Donor-specific tolerance in animals with CTTI versus general hyporesponsiveness in animals without CTTI. (A) Graft survival of BN (cervical) heart in recipients with or without CTTI. Recipients with CTTI rejected the BN heart rapidly (median survival time $=10 \pm 1.0$ days), whereas recipients without CTTI did not reject the third-party BN hearts. The BN control (no immunosuppression/thymectomy) shows rejection of BN hearts by LW rats. The syngeneic control shows lack of rejection of LW hearts by LW rats. Kaplan-Meier survival curve showed significant differences in the graft survival. (B) ISHLT grading showed a severe rejection of BN heart in animals with CTTI, whereas there was a significant reduction of rejection in recipients without CTTI compared with BN controls with no immunosuppression ( $n=3-5$ per group). Turkey test, ${ }^{* *} P<0.001$; NS, not significant $(P>0.05)$. (C) Representative scanned image of explanted BN heart graft at the time of rejection or designated time points posttransplantation. Both BN heart grafts from animals with CTTI (third panel) and allogeneic (second panel) showed severe mononuclear cell infiltration, whereas BN heart grafts from animals without CTTI (fourth panel) or animals with syngeneic CTTI (first panel) showed no sign of rejection. Images were adapted from whole slide scan. CTTI, cultured thymus tissue implantation; BN, Brown Norway; LW, Lewis; ISHLT, International Society for Heart and Lung Transplantation.

CTTI). BN hearts from recipients without CTTI did not show any increase in size (Supplemental Figure 6). Histological analysis (ISHLT grading) of explanted BN hearts from rats with CTTI (Figure 3C, third image) showed grade $3 \mathrm{R}$ rejection with significantly increased inflammatory cell infiltration compared with syngeneic controls (Figure 3C first image) or rats without CTTI (Figure 3C, fourth image).

Selective T cell infiltration in third-party hearts but not in DA hearts that shared the DA MHC of the CTTI. We used 2 conventional ways to define graft rejection in this rat heart transplantation model: heart beating/ cessation measurements and the ISHLT human grading system. The former is insensitive with respect to low-grade rejection, whereas the latter is insensitive with respect to high-grade rejection. Therefore, we evaluated inflammatory cell infiltration in DA hearts from 3 rats on day 180 and in BN hearts at the time of sacrifice at 7 to 8 months in 5 rats. As shown in Figure 4A, rats that were treated with $\mathrm{T}$ cell depletion, CTTI, and 4 months of CsA did not show an increased level of inflammatory cell infiltration in the DA hearts after T cell repopulation. On the other hand, the animals given CTTI showed massive inflammatory cell infiltration in the third-party cardiac allograft (BN heart) (Figure 4B). Rats without CTTI showed no infiltrates in the BN heart (Figure 4B). Finally, we evaluated T cell infiltration with IHC and confirmed a selective $\mathrm{T}$ cell infiltration in the BN (Figure 4C, third image), but not DA (Figure 4C, second panel), hearts of the animals with CTTI and a lack of T cell infiltration in both hearts of animals without CTTI (Supple- 
A Inflammatory cell infiltrate (DA heart)

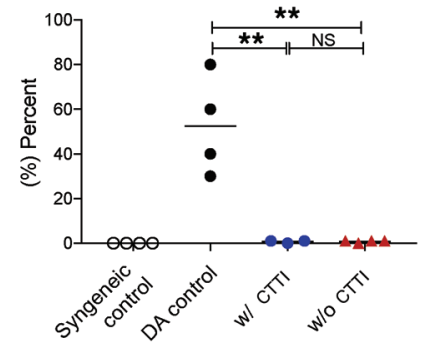

B Inflammatory cell infiltrate (BN heart)

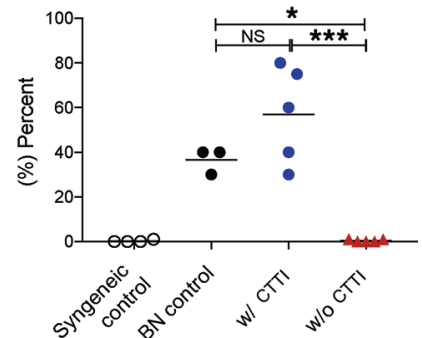

DA heart

BN heart

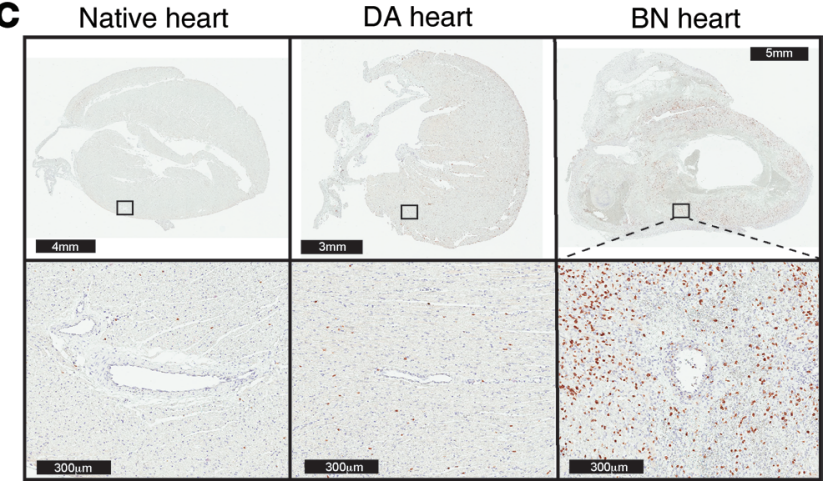

Figure 4. T cell infiltration (CD3 IHC) in LW, DA, and BN hearts. (A) Quantification of inflammatory cells in the primary abdominal DA cardiac allograft. DA grafts from animals both with and without CTTI showed no significantly reduced inflammatory cell infiltration (at POD180) compared with DA control grafts (in LW rats with no immunosuppression), showing a high level of graft infiltration of immune cells. (B) Quantification of inflammatory cells in secondary cervical BN cardiac allografts. Both the BN control grafts (in LW rats with no immunosuppression) and the BN grafts from recipients with CTTI showed significantly elevated inflammatory cell infiltration. The BN grafts from animals without CTTI showed significantly reduced inflammatory cell infiltration compared with BN control. (C) Heart allografts from DA and BN rats were harvested with native heart at the time of BN heart rejection. Grossly, native heart and DA heart (POD 196) did not show a dramatic increase of $T$ cells, whereas BN heart (POD14) showed a massive amount of T cells in recipients with CTTI. Images were adapted from whole slide scan. For panels $A$ and B, 3-5 animals per group were analyzed; Turkey test, ${ }^{*} P<0.05$; ${ }^{* *} P<0.01$; ${ }^{* * *} P<0.001$; NS, not significant $(P>0.05)$. LW, Lewis; DA, Dark Agouti; BN, Brown Norway; CTTI, cultured thymus tissue implantation.

mental Figure 7). These data confirm that the $\mathrm{T}$ cell infiltration occurs only in the third-party BN graft, but not in the graft sharing MHC (DA) with the transplanted thymus, possibly due to lack of $\mathrm{T}$ cell repertoire (by negative selection) against (DA heart) donor antigens.

Humoral response against donor antigens after thymus transplantation. After native LW thymectomy followed by LWxDA CTTI, we hypothesized that there would be a lack of T cell help for cognate B cell and downstream humoral response against the DA donor antigen in recipients. Therefore, we evaluated anti-donor antibody responses to determine whether the allogeneic $\mathrm{T}$ cell unresponsiveness was associated with humoral tolerance against donor DA MHC. We tested serially collected recipient serum samples and performed flow crossmatch with PBMCs from DA and BN rats. As expected, unmanipulated LW rats that received $\mathrm{DA}$ or $\mathrm{BN}$ heart transplants without immunosuppression developed antibody against their donors (DA or BN, respectively) (Figure 5A, first panel in each row). Animals with a syngeneic cardiac allograft did not produce antibody against either DA or BN MHC (data not shown). Interestingly, similar to $\mathrm{T}$ cell hyporesponsiveness, no anti-DA Ab was detected in animals with or without CTTI (Figure 5A, second and third panels of top row and Figure $5 \mathrm{~B})$. Anti-BN Ab was readily detected in animals with LWxDA CTTI but not in animals without CTTI $(P<0.01)$ (Figure 5A, second and third panels of bottom row, and Figure $5 \mathrm{C})$. Taken together, thymus cotransplantation resulted in specific tolerance to the allogeneic DA MHC expressed in the donor thymus and thus a long-term survival of the DA heart transplant via preventing the development of both the donor-specific anti-DA T cell repertoire and the donor-specific (DA-specific) humoral response. Immunocompetence was demonstrated in these rats by the rapid rejection of third-party $\mathrm{BN}$ hearts as well as alloantibody response against $\mathrm{BN}$ donor cells. 
A

DA control

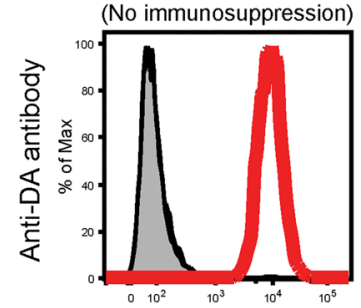

BN control

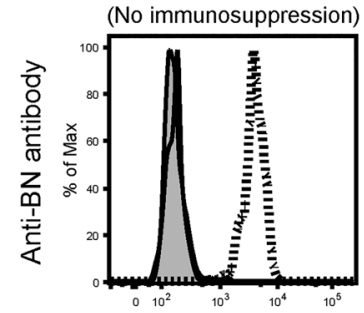

w/ CTT
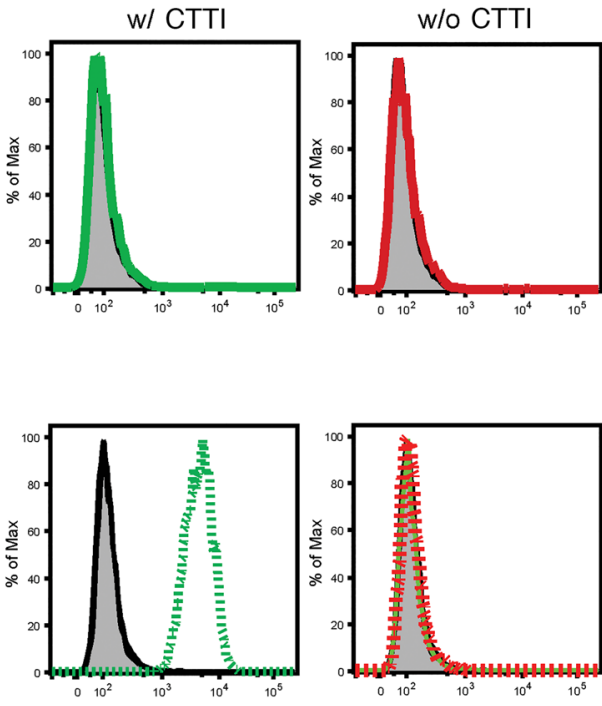

B
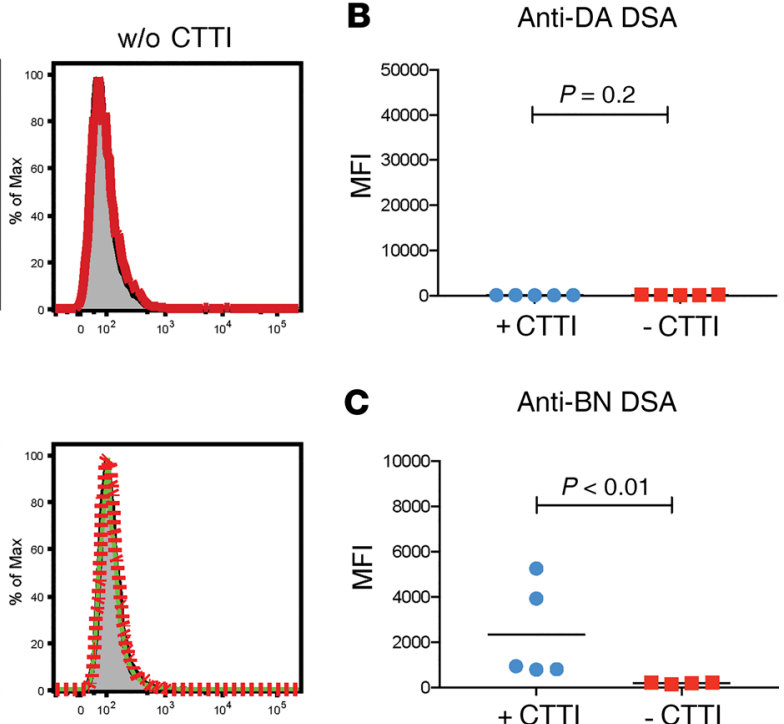

C

Anti-BN DSA

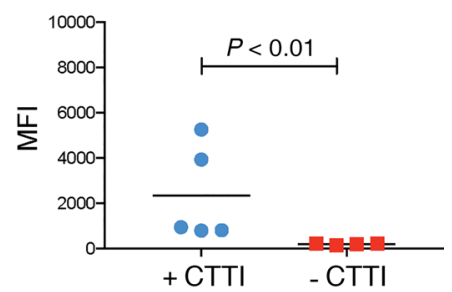

Figure 5. Donor-specific humoral tolerance with CTTI. (A) Representative histogram plots for posttransplant donor-specific alloantibody (anti-DA and anti-BN antibodies) measured by T cell flow crossmatch. LW recipients with or without CTTI did not generate any antibodies against DA antigen (A, second and third plots, respectively, in top row), whereas animals with CTTI were able to generate antibody against BN antigen (A, second panel, bottom row). Serum samples from LW recipients of DA heart transplantation without immunosuppression (A, first panel in top row) and from LW recipients of BN heart transplantation without immunosuppression (A, first panel in bottom row) were used as positive controls (DA control and BN control) for anti-DA or antiBN antibody, respectively. (B) Level of anti-DA antibody after primary DA heart transplantation. Serum samples from animals with CTTI and without CTTI after DA heart transplantation (POD 180) did not develop antibody against DA cells. (C) Levels of anti-BN antibody after secondary BN heart transplantation. Animals with CTTI showed significantly elevated anti-BN Abs, whereas animals without CTTI showed no antibodies against BN cells. A total 4 to 5 animals per group were analyzed; Student's $t$ test. CTTI, cultured thymus tissue implantation; DA, Dark Agouti; BN, Brown Norway; LW, Lewis.

\section{Discussion}

Achieving donor-specific immune tolerance remains the ultimate immunologic goal in transplantation. Most of the current approaches focus on controlling peripheral mature donor-reactive T cells by depletion (e.g., alemtuzumab, thymoglobulin, etc.) or suppression (e.g., calcineurin inhibitors, basiliximab, etc.) without targeting the production of alloreactive $\mathrm{T}$ cells in thymus. However, even with the dramatic advancement of immunosuppressive drugs and new immunomodulatory regimens, transplant tolerance has not yet been consistently achieved. The use of thymus tissue to promote or transfer immunologic tolerance such as by intrathymic injection of antigens has been investigated in many animal models (19-24). Over all, these studies have not been convincing due to the lack of histological evidence, proper controls, long-lasting efficacy, or translation into large animal models or humans. Our approach to achieve tolerance to donor antigens is to use donor CTTI to induce immune tolerance to allogeneic antigens. We hypothesize that tolerance to self is achieved because recipient dendritic cells induce apoptosis in thymocytes expressing $\mathrm{T}$ cell receptors that bind with high avidity/affinity to self-peptide/ self-MHC on recipient dendritic cells (25-27). In addition, tolerance to donor is achieved because TECs (28) present donor antigens directly, donor-peptide/donor-MHC (29-33) or indirectly via recipient DC, donor-peptide/recipient-MHC to thymocytes. Thus, TECs are a key component promoting donor-specific tolerance by deletion of thymocytes that bind strongly to recipient or donor MHC during allogeneic thymus transplantation.

Tolerance induction by CTTI is similar to tolerance induction via donor DC in hematopoietic stem cell transplantation $(34,35)$. A series of studies from Transplantation Biology Research Center showed the crucial role of thymus in tolerance induction (36) and tested thymus transplantation with tolerance induction in a large animal model $(9,37,38)$. In their series of studies, this group successfully used class II matched/class I mismatched donor (thymus and kidney or heart) as thymus composite tissues (thymokidney and thymoheart) with 12 days of CsA for transplant tolerance induction. This elegant concept of generating vascularized thymus before transplantation to induce tolerance would be difficult to translate to the clinic without using xenotransplantation. The authors stated that nonvascularized thymus did not induce tolerance in their model. More precisely, however, nonvascularized thymus that was not cultured did not engraft long term. We believe that the reason for lack of function of noncultured thymus is that there is so much cell death in freshly harvested thymus. The dying thymocytes release deoxyadenosine, 
which diffuses into neighboring thymocytes. The deoxyadenosine is then phosphorylated to deoxyadenosine monophosphate (dAMP), which is trapped in the cell. The dAMP is further phosphorylated into dATP, which inhibits ribonucleotide reductase and prevents DNA synthesis $(39,40)$. Thymocytes die in this environment. The culture system used in this report likely prevents buildup of deoxyadenosine. In particular, media is dripped on the thin thymus slices that are floating on sponges in a tissue culture dish. Every day the old medium is removed and new medium is dripped onto the tissue. The deoxyadenosine from the thymocytes is washed off the slices and thus does not lead to the toxic pathway described above.

The limitation of nonvascularized thymus transplantation can be overcome with a culture system as well as $\mathrm{T}$ cell depletion as shown in the present study. Experimental transplantation of allogeneic CTTI that retains TECs has been successfully applied to treat pediatric patients with complete DiGeorge anomaly who are born without thymus or thymus function $(17,41)$. Thymopoiesis has been documented by allograft biopsies and the presence of recipient naive T cells in the periphery $(13,18,42)$. Studies of children treated with investigational CTTI show tolerance to donor MHC by mixed lymphocyte reactions (14). In addition, the infants with complete DiGeorge anomaly, after CTTI, are able to control infections such as EpsteinBarr virus $(16,19)$. Based on these data in human infants, we hypothesize that implantation of allogeneic thymus expressing the MHC of a solid organ donor will result in donor-specific tolerance to both self and to the donor and also will retain functional $\mathrm{T}$ cells that will protect the recipient from infection.

In the present study, we largely adapted techniques from the clinical setting of CTTI. Rat thymus was cultured in a similar manner to that used for human thymus $(42,43)$. Cultured thymus tissue maintained the normal thymus structure but was partially thymocyte-depleted consistent with human data (Supplemental Figure 2). As shown in Figure 1C, and Supplemental Figure 1D, the CTTI was well engrafted under the kidney capsule with normal thymus structure. To test our tolerance hypothesis, we performed heterotopic DA heart transplantation together with $\mathrm{F} 1$ of LW and DA rat (F1[LW x DA]) CTTI in thymectomized, T cell-depleted LW rats (Figure 1A). The control group did not receive CTTI. Both groups of thymectomized LW rats developed circulating $\mathrm{T}$ cells after $\mathrm{T}$ cell depletion with anti-CD5 mAb followed by DA heart transplantation (Figure 1B). In the control group, the return of circulating $\mathrm{T}$ cells was likely due to $\mathrm{T}$ cell repopulation from the periphery (memory T cells), whereas the group with CTTI also had development of naive T cells in the thymus (Figure 1B). The CTTI in LW rats that were given DA heart transplants showed normal thymus structure with thymopoiesis (Figure 1C). There was no DA heart graft rejection in either group (with or without a F1[LWxDA] CTTI) when the heart beating was assessed by palpation (Figure 2A). We hypothesized that the $\mathrm{T}$ cells in the control group, although present in the circulation, were not functional. To test this, we used a third-party BN heart transplant to assess if either group could reject the third-party graft. The third-party BN heart allografts were promptly rejected from animals with CTTI but were not rejected by animals without CTTI. Thus, only the animals with CTTI had specific tolerance to donor MHC with immunocompetence demonstrated by the ability to reject allogeneic BN hearts.

Currently, long-term graft survival is often hindered by donor-specific antibodies, even with successful $\mathrm{T}$ cell control (44). We tested whether the donor-specific tolerance induced by cultured donor thymus tissue transplantation would have an impact on posttransplantation humoral responses. Our hypothesis was correct in that thymectomized, T cell-depleted LW rats given F1(LWxDA) CTTI did not develop donor-specific alloantibody (DSA) against the DA antigens in the abdominal DA heterotopic heart transplant (Figure 5A, top row, middle panel, and Figure 5B) but did make DSA against the $\mathrm{BN}$ heart as detected at the time of graft rejection (Figure 5A, bottom row, middle panel, and Figure $5 \mathrm{C})$. This suggests that allogeneic, thymus-induced, donor-specific tolerance might control anti-donor humoral response as well. Last, it is known that the major population of Foxp $3^{+}$Treg is generated in the thymus (45). Therefore, it is possible that Treg cells generated from CTTI could also provide additional regulation against donor Ag-reactive T cells.

We believe that the present study provides proof of concept for donor thymus cotransplantation with solid organs for tolerance induction. The patient group that would most benefit from the procedure is infants needing heart transplants. Because postnatal thymic tissue is present and could be removed from the deceased donor infant, and the recipient thymus is routinely removed from infants undergoing heart transplantation, no additional procedure aside from CTTI would be needed to transfer this approach to the clinic. Overall, these results support the use of CTTI for the tolerance induction in organ transplantation; however, it remains to be tested as to how efficiently transplanted cultured thymus tissue will develop donor-specific tolerance in an immunocompetent large animal model with clinically relevant immunosuppressive regimens before moving into the clinic. 


\section{Methods}

Animal models. LW (RT-1) and BN (RT- $\left.1^{\mathrm{n}}\right)$ rats were purchased from Charles River. DA (RT-1 ${ }^{\text {av1 }}$ ) rats were purchased from Envigo. F1(LWxDA; RT-1/av1) were bred by protocol staff at the Duke Breeding Core Division of Laboratory Animal Resources facility. LW recipients received thymectomies as previously described (46). Briefly, the submandibular glands and sternohyoid muscle were separated with blunt forceps to expose the overlying the trachea. A $1-$ to $1.5-\mathrm{cm}$ incision was made in the sternal manubrium. A 7 -cm alms-type retractor was used to retract the manubrium and the 2 halves of the sternohyoid muscles to expose the thymus. The thymus was grasped with blunt forceps and extracted. The cut ends of the sternum were closed with a single 3 to $4-0$ silk suture. Two drops of bupivacaine $(2.5 \mathrm{mg} / \mathrm{mL})$ were applied on the incision and the outer layer of skin was closed with 3- or 4 9-mm wound clips. All thymectomized rats were maintained on a diet containing Septra (PMI Nutrition International). To induce T cell depletion in vivo, 1 mg anti-CD5 mAb (OX19, BioXCell) was intraperitoneally administered on days 0, 5, and 10 after thymectomy and suppression with $0.25 \mathrm{mg} / \mathrm{kg} / \mathrm{d}$ cyclosporine pump from day 0 (heart transplant and CTTI time point) to 4 months with respect to heart transplantation.

In vitro thymus culture and CTTI. Thymus from 3-day-old neonatal F1 (LWxDA) rat pups were harvested sterilely, cut into 4 pieces along the longitude natural seam, and transferred onto sterile nitrocellulose filters (MilliporeSigma) in a tissue culture dish with thymus organ medium (TOM) medium. Thymus tissue was cultured in a $\mathrm{CO}_{2}$ incubator with $5 \% \mathrm{CO}_{2}$ at $37^{\circ} \mathrm{C}$ for the desired length of time (5 to 7 days). The medium was changed daily. The TOM comprised Ham's F-12 (Life Technologies) at 86.5\%; HEPES (Life Technologies) at $25 \mathrm{Mm}$; L-glutamine (Life Technologies) at $2 \mathrm{Mm}$; FBS (Life Technologies) at 10\%; and Pen Strep (Life Technologies) used at $1 \times$. On the day of transplantation, the thymus pieces were rinsed with fresh medium and transplanted under the kidney capsule of a LW rat with one secure suture (10-0 monofilament). All manipulations took place under sterile conditions in a biological safety cabinet.

Abdominal and cervical heart transplantations. Full MHC-mismatched DA (RT-1 $\left.{ }^{\text {av1 }}\right)$ donor hearts were transplanted into thymectomized LW (RT-1 $1^{1}$ ) recipients. Abdominal heart transplantation was performed using a modified technique of the methods described by Schmid et al. (47). Briefly, the donor heart was transplanted into the abdominal cavity of the recipient after a short period of cold ischemia in Euro-Collins solution. The donor pulmonary artery and aorta were anastomosed to the recipient inferior vena cava and descending aorta with an end-side fashion as the inflow and outflow vessels for circulation, using running 9/0 nonabsorbable monofilament sutures. CsA was given via the osmotic pump (model 2ML4, Alzet). The pump was loaded sterilely and surgically inserted subcutaneously to mid-dorsal area of recipients. The osmotic pump was replaced every month for 4 months. For full MHC-mismatched BN $\left(\mathrm{RT}-1^{\mathrm{n}}\right)$ third-party heart transplantation to the DA heart bearing LW recipients, we used cervical vascularized heart transplantation described by Heron in a modified fashion (48). Briefly, the third-party heart was transplanted into the right side of cervical area via a longitudinal incision from submaxilla to the xiphoid. The donor pulmonary artery and external jugular vein were anastomosed end to end and the aorta was anastomosed to the right common carotid artery by cuffing technique. The grafts were monitored by daily palpation and later confirmed by laparotomy at the time of sacrifice. Animals were sacrificed on the day of rejection of the graft (cessation of beating) or a designated time point.

Flow cytometric analysis and monitoring DSA. Peripheral blood was obtained from the cranial vena cava and stained with antibodies. To analyze naive and recent thymic emigrants, we used the combination of anti-Rat CD3-APC (BD Biosciences); anti-Rat CD4-APC-Cy7 (BioLegend); anti-Rat CD8a-V450 (BD Biosciences); anti-Rat CD45-PE-Cy7 (BD Biosciences); anti-Rat CD45RC- PE (BD Biosciences); antiRat CD62L-FITC (BD Biosciences); and anti-Rat CD90-BV-510 (BioLegend). To assess percentages of T, B, and NK cells, we used the combination of anti-Rat TCR-FITC (BD); anti-Rat CD4-APC-Cy7 (BioLegend); anti-Rat CD8a-V450 (BD); anti-Rat CD45-PE-Cy7 (BD); anti-Rat CD45RA-PE (Invitrogen); and anti-Rat NKR-P1A-APC (Invitrogen). For host versus donor discrimination, we used the combination of anti-Rat TCR-APC (BioLegend), anti-Rat CD45-PE-Cy7 (BD Biosciences); and MHC class I RT1Aa (Santa Cruz Biotechnology). We also used a secondary goat anti-mouse IgG (Invitrogen) for the nonconjugated MHC class I RT1Aa. DSA was assessed by flow crossmatch from serially collected recipient serum samples with DA donor or BN third-party rats. FITC-conjugated pan-rat immunoglobulin antibody was added to the samples and incubated after washing. The T cells were stained with APC-conjugated antiCD3. Samples were analyzed on a LSR-Fortessa (Beckman Coulter).

Histology, IHC, and morphological analysis. All cultured thymus and CTTI samples from under the kidney capsule were frozen in optimal cutting compound (Tissue Tek). Control thymus tissue was obtained 
from newborn to 5-day-old rat pups. Sections (4-5 mm) were stained for CD3 (polyclonal, catalog A0452, Dako), Ki-67 (SP6, clone, catalog RM-9106-S1, Thermo Fisher Scientific), and CK (polyclonal, cata$\log 180059$, Invitrogen). IHC images were obtained using an Olympus Vanox AH-3 Microscope of the Olympus DP-70 Digital Camera System. The explanted hearts underwent serial sectioning (5 $\mu \mathrm{m})$ from the midventricular level to the base. H\&E stains were performed for routine examination and grading of rejection. Graft-infiltrating T cells were evaluated with polyclonal anti-CD3 (Dako) staining. Whole slides of grafts were scanned with an Aperio ScanScope XT (Aperio Technologies).

Statistics. Experimental results were analyzed by a GraphPad Prism software version 7.0 and SAS version 9.4. The log-rank test was used for differences in graft survival; 2-tailed Student's $t$ test was used for comparisons between 2 groups; and Turkey test was used for multiple group comparison. All the data were presented as mean \pm SD. $P$ values of less than 0.05 were considered statistically significant.

Study approval. All rats were used and maintained in accordance with the guidelines of and in compliance of Duke Institutional Animal Research Ethics Committee (IACUC), which approved these studies.

\section{Author contributions}

JK designed experiments, performed surgical procedures, conducted in vitro experiments, analyzed, interpreted data, and prepared the manuscript. JL designed experiments, cared for experimental animals, conducted in vitro experiments (including flow cytometry), and prepared the manuscript. CR participated in surgical procedures (thymectomy and phlebotomy) and cared for experimental animals. JP participated in surgical procedures (heart transplantation). ABF interpreted data (pathologist). MK participated in analyzing data (biostatistician). JWT edited the manuscript. SJK interpreted data and edited the manuscript. ADK conceived of experimental design and edited the manuscript. MLM conceived of experimental design, interpreted data, and prepared the manuscript.

\section{Acknowledgments}

This work was supported by a Duke Chancellor's Discovery Grant (to MLM), Duke Surgery internal grant (to MLM), Duke Transplantation Center pilot grant (to JK), American Heart Association (AHA)/Enduring Heart Foundation Research Award 15SDG25710165 (to JK), and a grant from Enzyvant (to MLM). The authors thank Duke Laboratory Animal Resources for veterinary support and Stephanie Hasapis and Heather Daniels for their expert assistance. We acknowledge Li-Wen Li (Duke University) for preliminary work.

Address correspondence to: M. Louise Markert, Box 3068, DUMC, Durham, North Carolina 27710, USA. Phone: 919.684.6263; Email: m.markert@duke.edu.

1. Owen RD. Immunogenetic consequences of vascular anastomoses between bovine twins. Science. 1945;102(2651):400-401.

2. Billingham RE, Brent L, Medawar PB. Actively acquired tolerance of foreign cells. Nature. 1953;172(4379):603-606.

3. Adkins B, Leclerc C, Marshall-Clarke S. Neonatal adaptive immunity comes of age. Nat Rev Immunol. 2004;4(7):553-564

4. Garcia AM, Fadel SA, Cao S, Sarzotti M. T cell immunity in neonates. Immunol Res. 2000;22(2-3):177-190.

5. Hall BM, Dorsch S, Roser B. The cellular basis of allograft rejection in vivo. II. The nature of memory cells mediating second set heart graft rejection. J Exp Med. 1978;148(4):890-902.

6. Hall BM, Dorsch S, Roser B. The cellular basis of allograft rejection in vivo. I. The cellular requirements for first-set rejection of heart grafts. J Exp Med. 1978;148(4):878-889.

7. Brent L, Brooks CG, Medawar PB, Simpson E. Transplantation tolerance. Br Med Bull. 1976;32(2):100.

8. Streilein JW. Neonatal tolerance of H-2 alloantigens. Procuring graft acceptance the "old-fashioned" way. Transplantation. 1991;52(1):1-10.

9. Yamada K, et al. Thymic transplantation in miniature swine. II. Induction of tolerance by transplantation of composite thymokidneys to thymectomized recipients. J Immunol. 2000;164(6):3079-3086.

10. Menard MT, et al. Composite "thymoheart" transplantation improves cardiac allograft survival. Am J Transplant. 2004;4(1):79-86.

11. Johnston DR, et al. Heart and en-bloc thymus transplantation in miniature swine. J Thorac Cardiovasc Surg. 2005;130(2):554-559

12. Nobori S, et al. Long-term acceptance of fully allogeneic cardiac grafts by cotransplantation of vascularized thymus in miniature swine. Transplantation. 2006;81(1):26-35.

13. Markert ML, Devlin BH, McCarthy EA. Thymus transplantation. Clin Immunol. 2010;135(2):236-246.

14. Chinn IK, Devlin BH, Li YJ, Markert ML. Long-term tolerance to allogeneic thymus transplants in complete DiGeorge anomaly. Clin Immunol. 2008;126(3):277-281.

15. Chinn IK, et al. Mechanisms of tolerance to parental parathyroid tissue when combined with human allogeneic thymus transplantation. J Allergy Clin Immunol. 2010;126(4):814-820.e8.

16. Markert ML. In: Sullivan KE, Stiehm ER, eds. Stiehm's Immune Deficiences. Cambridge, MA: Academic Press; 2014:1059-1067.

17. Markert ML, et al. Postnatal thymus transplantation with immunosuppression as treatment for DiGeorge syndrome. Blood. 
2004;104(8):2574-2581.

18. Markert ML, et al. Use of allograft biopsies to assess thymopoiesis after thymus transplantation. J Immunol. 2008;180(9):6354-6364

19. Isakovic K, Smith SB, Waksman BH. Immunologic tolerance in thymectomized, irradiated rats grafted with thymus from tolerant donors. Science. 1965;148(3675):1333-1335.

20. Posselt AM, Barker CF, Tomaszewski JE, Markmann JF, Choti MA, Naji A. Induction of donor-specific unresponsiveness by intrathymic islet transplantation. Science. 1990;249(4974):1293-1295.

21. Remuzzi G, Rossini M, Imberti O, Perico N. Kidney graft survival in rats without immunosuppressants after intrathymic glomerular transplantation. Lancet. 1991;337(8744):750-752.

22. Chowdhury NC, et al. Acquired systemic tolerance to rat cardiac allografts induced by intrathymic inoculation of synthetic polymorphic MHC class I allopeptides. Transplantation. 1996;62(12):1878-1882.

23. Gottrand G, Taleb K, Ragon I, Bergot AS, Goldstein JD, Marodon G. Intrathymic injection of lentiviral vector curtails the immune response in the periphery of normal mice. J Gene Med. 2012;14(2):90-99.

24. Chu Q, et al. Induction of immune tolerance to a therapeutic protein by intrathymic gene delivery. Mol Ther. 2010;18(12):2146-2154

25. Ashton-Rickardt PG, Van Kaer L, Schumacher TN, Ploegh HL, Tonegawa S. Peptide contributes to the specificity of positive selection of CD8+ T cells in the thymus. Cell. 1993;73(5):1041-1049.

26. Hogquist KA, Gavin MA, Bevan MJ. Positive selection of CD8+ T cells induced by major histocompatibility complex binding peptides in fetal thymic organ culture. J Exp Med. 1993;177(5):1469-1473.

27. Brocker T, Riedinger M, Karjalainen K. Targeted expression of major histocompatibility complex (MHC) class II molecules demonstrates that dendritic cells can induce negative but not positive selection of thymocytes in vivo. J Exp Med. 1997;185(3):541-550.

28. Sekai M, Hamazaki Y, Minato N. Medullary thymic epithelial stem cells maintain a functional thymus to ensure lifelong central T cell tolerance. Immunity. 2014;41(5):753-761.

29. Derbinski J, Schulte A, Kyewski B, Klein L. Promiscuous gene expression in medullary thymic epithelial cells mirrors the peripheral self. Nat Immunol. 2001;2(11):1032-1039.

30. Derbinski J, et al. Promiscuous gene expression in thymic epithelial cells is regulated at multiple levels. J Exp Med. 2005;202(1):33-45.

31. Klein L, Kyewski B. "Promiscuous" expression of tissue antigens in the thymus: a key to T-cell tolerance and autoimmunity? J Mol Med. 2000;78(9):483-494.

32. Magalhães DA, et al. Promiscuous gene expression in the thymus: the root of central tolerance. Clin Dev Immunol. 2006;13(2-4):81-99.

33. Spitzweg C, Joba W, Heufelder AE. Expression of thyroid-related genes in human thymus. Thyroid. 1999;9(2):133-141.

34. Sharabi Y, Sachs DH. Mixed chimerism and permanent specific transplantation tolerance induced by a nonlethal preparative regimen. J Exp Med. 1989;169(2):493-502.

35. Manilay JO, Pearson DA, Sergio JJ, Swenson KG, Sykes M. Intrathymic deletion of alloreactive T cells in mixed bone marrow chimeras prepared with a nonmyeloablative conditioning regimen. Transplantation. 1998;66(1):96-102.

36. Yamada K, et al. Role of the thymus in transplantation tolerance in miniature swine. I. Requirement of the thymus for rapid and stable induction of tolerance to class I-mismatched renal allografts. J Exp Med. 1997;186(4):497-506.

37. Yamada K, et al. Thymic transplantation in miniature swine: III. Induction of tolerance by transplantation of composite thymokidneys across fully major histocompatibility complex-mismatched barriers. Transplantation. 2003;76(3):530-536.

38. Nobori S, et al. Thymic rejuvenation and the induction of tolerance by adult thymic grafts. Proc Natl Acad Sci U S A. 2006;103(50):19081-19086.

39. Webster AD. Metabolic defects in immunodeficiency diseases. Clin Exp Immunol. 1982;49(1):1-10.

40. Mitchell BS, Kelley WN. Purinogenic immunodeficiency diseases: clinical features and molecular mechanisms. Ann Intern Med. 1980;92(6):826-831.

41. Markert ML, et al. Transplantation of thymus tissue in complete DiGeorge syndrome. NEngl J Med. 1999;341(16):1180-1189.

42. Markert ML, et al. Review of 54 patients with complete DiGeorge anomaly enrolled in protocols for thymus transplantation: outcome of 44 consecutive transplants. Blood. 2007;109(10):4539-4547.

43. Markert ML, et al. Thymus transplantation in complete DiGeorge syndrome: immunologic and safety evaluations in 12 patients. Blood. 2003;102(3):1121-1130.

44. Terasaki PI. Humoral theory of transplantation. Am J Transplant. 2003;3(6):665-673.

45. Shevach EM, Thornton AM. tTregs, pTregs, and iTregs: similarities and differences. Immunol Rev. 2014;259(1):88-102.

46. Rendell VR, Giamberardino C, Li J, Markert ML, Brennan TV. Complete thymectomy in adult rats with non-invasive endotracheal intubation. J Vis Exp. 2014;(94):52152.

47. Schmid C, Binder J, Heemann U, Tilney NL. Successful heterotopic heart transplantation in rat. Microsurgery. 1994;15(4):279-281.

48. Heron I. A technique for accessory cervical heart transplantation in rabbits and rats. Acta Pathol Microbiol Scand A.

1971;79(4):366-372. 